\title{
National Thalassaemia eRegistry: using IT for effective health resource management
}

\author{
Dr. S. Peiris \\ Ministry of Health, Sri Lanka. \\ Dr. R. Hewapathirana \\ Post graduate Institute of Medicine, University of Colombo \\ Dr. R. Rampatige \\ Post graduate Institute of Medicine, University of Colombo \\ Dr. A. Hanifa \\ Postgraduate Institute of Medicine, University of Colombo, Sri Lanka \\ Contact e-mail address: sharikapeiris@gmail.com \\ eHealth Sri Lanka 2010,1(suppl.1):S15 \\ DOI: http://dx.doi.org/10.4038/sljbmi.v1i0.3550
}

Only the Abstract is available

\begin{abstract}
Thalassaemia is an inherited autosomal recessive blood disorder, resulting in reduced rate of synthesis of one of the haemoglobin chains, causing chromic anaemia. Majority of the thalassaemia patients are managed by the government hospitals at some stage of the disease. Disease is diagnosed usually after six months of age with anemia requiring monthly blood transfusion leads to accumulation of excessive iron and iron overload in the heart, liver and other vital organs. At present the data on these patients are recorded at the ward level of each institution without uniformity and reporting to the central level is also found to be incomplete. There is no proper way to manage the data flow of Thalassaemia patients making it unrealistic to reflect the data reported at hospital level to district, provincial and national level. Lack of consistent and timely information flow on distribution and management of Thalassaemia patients in Sri Lanka is a major draw back in planning adequate prevention and control strategies and resource allocation. The Thalassaemia eRegisstry developed focused on optimisation of patient management data, assisting the diagnosis and clinical decision making process and observing the overall management process helping the administrative layer to easy decision making and policy development. Number of patients island-wide, number of newly diagnosed cases and deaths, number of patients on different treatment methods, average hospital stay and cost of each Thalassamia admission are some of the key factors focused in the eRegistry. Special services needed for the improvement of quality of life, costing of the service, identification of transfusion and drug related issues and patients needing special attention are among the expected outcomes. The project is currently piloted at Kurunegala and Anuradhapura Thalassaemia Centres.
\end{abstract}

Keywords - Thalassaemia eRegisstry, patient management data 